\title{
Revisions to the Animal Welfare Inspection Guide
}

\author{
B. Taylor Bennett, Andrew D. Cardon \& Matthew R. Bailey
}

On 2 March 2015, the US Department of Agriculture (USDA) announced that it had revised its Animal Welfare Inspection Guide $^{1}$ (AWIG) to improve the oversight of research facilities. The revision was made in response to the report from the USDA's Office of Inspector General (OIG) discussed in this column in April 2015 (ref. 2). Revisions were made to Chapter 7, Chapter 4 , page 25 (related to the instructions for inspecting research facilities that have not in engaged in regulated activities for the past two years) and the guidance documents for completing the Annual Report (Appendix A). Numerous changes were made to Chapter 7 on 'Research Facility Inspection-IACUC Requirements', four of which were highlighted in the announcement and are discussed below.

\section{Reporting exceptions and exemptions}

On page 26, the language related to reporting exceptions and exemptions was changed to indicate that where the regulations or standards provide the IACUC with a mechanism for approving a specific activity-either as an exception or exemption to the regulations and standards-an explanation for the approval should not be included in the annual report. This includes approval of multiple major operative procedures under one protocol, exemptions to the plans for exercise for dogs or environmental enhancement of nonhuman primates, shortterm food and water restrictions and deviations from the methods of euthanasia, as defined in the Animal Welfare Act (AWA) Regulations.

Guidance related to significant changes On page 27, guidance is provided to the veterinary medical officers (VMOs) to align the USDA inspection process with the recently released Office of Laboratory Animal

National Association for Biomedical Research, Washington, DC.
Welfare guidance on handling significant changes to protocols ${ }^{3}$. Taking advantage of this change through the development of standard operating procedures (SOPs), guidance documents and formularies can reduce the administrative burden for the IACUC and the investigator. Implementation of these changes and development of a system to keep protocols current with the changes made should minimize citations resulting from incongruences between an ongoing activity and an approved protocol.

\section{Review of protocols during inspections} Revisions on page 29 address the OIG recommendation that USDA should provide guidance to VMOs on how to select and review various types of protocols. An issue of concern addressed in our previous article concerning VMOs documenting and maintaining a record of the protocols reviewed and the rationale for selecting them was that such records would be available under the federal Freedom of Information Act. This issue has been effectively addressed. The Research Facility Protocol Selection Worksheet can be found on page 59. It contains five categories of reasons for selecting a protocol and a column for indicating how many protocols were selected for each of those categories. The individual protocols are not identified.

\section{Inclusion of non-farm animals}

On page 36, changes were made to include non-farm animals such as rabbits, guinea pigs and hamsters used for the development or testing of agricultural products or production of serum. This indicates that they are covered by the regulations and subject to inspection. Personnel at facilities that use non-farm animals to develop and test products for farm animals should carefully read this revised section.

In addition to the four changes highlighted in the announcement, a few additional key changes should be reviewed. Page 8 now contains a Notice with a definition of "A quorum", which has been revised to accurately reflect the definition in the AWA. Page 11 contains a statement emphasizing the fact that the AWA does not authorize the IACUC to dictate to researchers how to conduct their research. Following a list of IACUC functions on page 17 is a new Notice, which reads, "There is no requirement for every SOP to be reviewed every six months. The IACUC may determine a reasonable schedule for review of SOPs." On page 20, six bulleted items have been included as examples of the type of rationale the USDA is looking for in the protocol section on determining the number of animals to be used. On page 24 , there is expanded guidance under the subsection on 'Major Operative Procedures', and on page 25, there is a new Notice on laparoscopic surgery. On page 41, the subsection on 'Continuing Review' has been expanded to include more definitive information on the timing of the review, how the review should be conducted and what should be considered in the review. There is a new section on 'Contracted Research or Projects that Involve Multiple Registrants' on page 43, which describes the requirements for assigning responsibilities for such arrangements.

The revisions to Chapter 7 of the AWIG have also addressed some of the inconsistencies in the previous version, which should make it more user-friendly for those responsible for managing the inspection process.

1. United States Department of Agriculture. Animal Welfare Inspection Guide (US Department of Agriculture, Riverdale, MD, 2015).

2. Bennett, B.T., Bailey, M.R. \& Cardon, A.D. USDA Inspector General's report on oversight of research facilities. Lab Anim. (NY) 44, 127-128 (2015).

3. National Institutes of Health. Office of Laboratory Animal Welfare-Significant Changes to Animal Activities (National Institutes of Health, Bethesda, MD, 2014). <http://grants. nih.gov/grants/olaw/significant_changes.htm> 